\title{
Josephson current in $d$-wave superconductor junctions with ferromagnetic insulator
}

\author{
Yan-Hua Liao, Meng Yang, Chang Ma, and Yu-Bin Cao \\ School of Mathematics and Physics, Huangshi Institute of Technology \\ Huangshi 435100, People's Republic of China \\ E-mail: xiaoyu35051@sina.com
}

Received September 18, 2011, revised December 15, 2011

\begin{abstract}
We investigate the temperature dependence of the critical current and current-phase relation by taking into account the ferromagnetic scattering effect at interface in a $d$-wave superconductor (S)/ferromagnetic insulator layer (FI)/d-wave superconductor (S) junction. It is shown that both the barrier scattering and the roughness scattering at the interface always suppress the Andreev reflection. The Josephson critical currents depend to a great extent on the effective exchange field of the interface and the crystal orientation of the $d$-wave superconductor. The exchange field can lead to the change of the junction from 0 to $\pi$ states and the alteration of the oscillation periods. It can also enhance the Josephson critical current in the junction under certain conditions.

PACS: 74.72.-h Cuprate superconductors;

75.70.Cn Magnetic properties of interfaces (multilayers, superlattices, heterostructures).
\end{abstract}

Keywords: high- $T_{c}$ superconductors, ferromagnetic insulator, Josephson current.

\section{Introduction}

The advance of Josephson effect has received a longstanding interest in understanding unusual behavior in superconductor/insulator/superconductor junction [1], it has been an interesting subject in condensed matter physics and studied in various situations [2-4]. Recently, superconductivity in the $\mathrm{Cu}-\mathrm{O}$ high- $T_{c}$ superconductors is attracting much attention, particularly in view of the recent many experimental and theoretical are focus on the $\mathrm{La}_{1.88} \mathrm{Sr}_{0.12} \mathrm{CuO}_{4}$ and $\mathrm{Sm}_{1.85} \mathrm{Ce}_{0.15} \mathrm{CuO}_{4-\delta}$ again $[5,6]$. Some theoretical and experimental suggest $d_{x^{2}-y^{2}}$-wave symmetry in the pair potential in these material [7], where $a, b$ are defined as the $\mathrm{CuO}_{2}$ plane of $a$ and $b$ axes. Using superconducting quantum-interference devices (SQUID), Josephson junctions [8] show results which are consistent with $d_{x^{2}-y^{2}}$-wave symmetry of the pair potentials.

Josephson junctions are building elements of many electronic and electromagnetic mesoscopic structures, which are proved to be promising elements of superconducting classical and quantum circuits $[9,10]$. In particular, junctions consisting of superconducting and ferromagnetic materials have been widely used as novel functional devices [11]. The ferromagnet $(\mathrm{F})$ has been introduced to the Josephson tunnel structure, giving rise to some new physical effects. As an example, if very thin insulating layer in a S/I/S junc- tion is replaced by thin ferromagnetic layer (with I denoting an insulating layer), a new type of $\mathrm{S} / \mathrm{F} / \mathrm{S}$ Josephson junction is formed [12]. Bergeret et al. [13] proposed a Josephson tunnel junction of two F/S bilayers separated by a thin insulating film. On the assumption that a thin $\mathrm{F} / \mathrm{S}$ bilayer is equivalent to a homogeneous ferromagnetic superconductor (FS), the $\mathrm{S} / \mathrm{F} / \mathrm{I} / \mathrm{F} / \mathrm{S}$ structure may be simplified as a FS/FS junction. They found that the presence of an exchange field may increase the critical current in the FS/FS junction. By calculating a matrix current within the circuit transport theory, Yokoyama et al. [14] indicated that the Josephson current can be enhanced by the exchange field in $s$-wave superconductor/diffusive ferromagnet $p$-wave superconductor junctions, there have been some similar phenomena with FS/I/FS junctions. Samal et al. [15] have also systematically studied the influence of the ferromagnetic layer on the magnetic and transport properties in superconductor/ferromagnet bilayer. It is known that the critical current of SFS junctions changes its sign and the ground state from 0 to $\pi$ as a function of the ferromagnetic layer [16], meanwhile, Rahnavard et al. [17] found the spin current associated with the $0-\pi$ transition in a clean superconductor/normal metal/superconductor Josephson junction with triplet $f$-wave superconductors. There have been several theoretical doing works [18-20]. 
In addition to these theory, several theories of Josephson junctions contain unconventional superconductors have been presented which take into account the anisotropy of the pair potential $[21,22]$. Although all of them have succeeded in revealing some aspects of the Josephson effect, one more essential effect, the temperature dependence of the critical current and current-phase relation by taking into account the ferromagnetic scattering effect at interface in a $d$-wave superconductor ( $\mathrm{S}$ )/ferromagnetic insulator layer (FI) $/ d$-wave superconductor (S) $(d / \mathrm{FI} / d)$ junction, has not been devoted introduced yet. Since the formation of the zero-energy states (ZES) is a general phenomenon in unconventional superconductor junctions [23,24], the zerobias conductance peak (ZBCP) is also confirmed in $d$-wave superconductor junctions $[25,26]$. Therefore, the Josephson effects of $d$-wave superconductor junction with a ferromagnetic insulator may be significantly modified by these interference effects.

In this article, we first assume $\mathrm{La}_{1.88} \mathrm{Sr}_{0.12} \mathrm{CuO}_{4}$ and $\mathrm{Sm}_{1.85} \mathrm{Ce}_{0.15} \mathrm{CuO}_{4-\delta}$ have $d$-wave pairing state with $d_{x^{2}-y^{2}}$ symmetry, then study theoretically Josephson currents between two $d$-wave superconductors through ferromagnetic insulator layer, which is calculated from the Andreev reflection coefficients by an extended Furusaki and Tsukada (FT) approach [27]. By comparing the $\pi / 4-\pi / 4$ junction with the $0-\pi / 4$ junction, we have reported [28] that abrupt current and oscillation periods correlate with the crystal orientations of the superconductors on the two sides and barrier strength in a $d / \mathrm{I} / d$ junction. For more details on the numerical and analytical approaches, we refer the reader to Ref. 28. The influence of ferromagnetic scattering on Josephson current in a $d / \mathrm{FI} / d$ junction is mainly presented in this paper and the result exhibits that superconducting junction with a ferromagnetic insulator shows more physical phenomena than which one with a normal metal insulator.

\section{The model and calculation methods}

The model of the junction is shown in Fig. 1, we consider a two-dimensional $d / \mathrm{FI} / d$ junction structure, in which the left and right electrodes are made of $d$-wave $\mathrm{S}$, and they are separated by very thin ferromagnetic insulating layer. The thin ferromagnetic insulating layers at $x=0$ can be modeled to be a $\delta$-type barrier potential $V(x)=U \delta(x)$. In the presence of interface roughness, the interface potential may be modeled $[29,30]$,

$$
U \delta(x)=\left(U_{0} \hat{I}-i P \hat{\tau}_{3}+\eta_{\sigma} U_{h} \hat{\tau}_{3}\right) \delta(x) .
$$

Where $\hat{I}$ is the unit matrix and $\hat{\tau}_{3}$ is the Pauli matrix. In this effective potential, $U_{0}$ indicates the barrier strength, $P$ describes the scattering effect during tunneling through the rough barrier, $U_{h}$ is the exchange potential at interface, and $\bar{\sigma}$ is the spin opposite to $\sigma$ with $\uparrow$ and $\downarrow$, $\eta_{\sigma}=1$ for $\sigma=\uparrow$ and $\eta_{\sigma}=-1$ for $\sigma=\downarrow$. As in the pre-

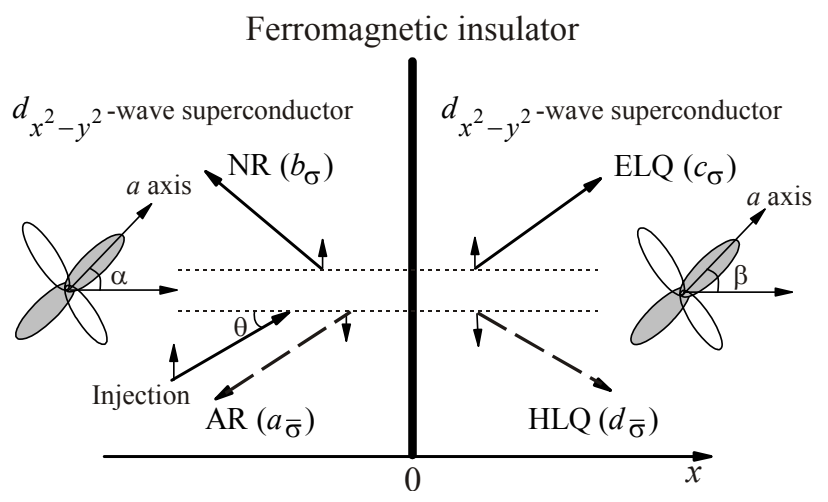

Fig. 1. Schematic illustration of the reflection and the transmission process of the quasiparticle for a $d / \mathrm{FI} / d$ junction.

vious works, we neglect for simplicity the self-consistency of spatial distribution of the pair potential in superconductor and take them as a step function $\Delta(x)=\Delta(\theta) \times$ $\times\left[\mathrm{e}^{i \phi_{L}} \Theta(-x)+\mathrm{e}^{i \phi_{R}} \Theta(x)\right]$, where $\Delta(\theta)$ is the pair potential for a $d$-wave $\mathrm{S}$, with $\theta$ is the angle between the quasiparticle momentum and the interface normal at $x=0$. The $\phi_{L}$ and $\phi_{R}$ stand for the macroscopic phase of the left and right superconductor, respectively. $\Delta(\theta)$ is described as

$$
\Delta(\theta)=\Delta_{ \pm}=\Delta_{d 0} \cos (2 \theta \mp 2 \alpha(\beta))
$$

for $d_{x^{2}-y^{2}}$ and

$$
\Delta(\theta)=\Delta_{ \pm}=\Delta_{d 0} \sin (2 \theta \mp 2 \alpha(\beta))
$$

for $d_{x y}$. Here $\Delta_{+}\left(\Delta_{-}\right)$corresponds to the pair potentials for electronlike (holelike) quasiparticles; $\alpha, \beta$ is the angle between the $a$ axis of the $d$-wave S crystal and the interface normal, and $\Delta_{d 0}$ is the order parameter of the $d$-wave superconductor at $T=0 \mathrm{~K}$, as shown in Fig. 1 .

We adopt the Bogoliubov-de Gennes (BdG) approach [31] to study the transport coefficients of the quasiparticles in the $d$-wave $\mathrm{S} / \mathrm{FI} / d$-wave $\mathrm{S}$ structures. In the absence of spin-flip scattering, the four-component BdG equations may be decoupled into two sets of two-component equations: one for spin-up electronlike and spin-down holelike quasiparticle wave functions $\left(u_{\uparrow}, v_{\downarrow}\right)$, the other for $\left(u_{\downarrow}, v_{\uparrow}\right)$. The decoupled BdG equation has the form

$$
\left(\begin{array}{cc}
H_{0}(r) & \Delta(\theta) \\
\Delta^{*}(\theta) & -H_{0}^{*}(r)
\end{array}\right)\left(\begin{array}{l}
u_{\sigma}(r) \\
v_{\bar{\sigma}}(r)
\end{array}\right)=E\left(\begin{array}{l}
u_{\sigma}(r) \\
v_{\bar{\sigma}}(r)
\end{array}\right),
$$

where $H_{0}(r)=-\hbar^{2} \nabla_{r}^{2} / 2 m+U(r)-E_{F}$ with $U(r)$ the usual static potential, and the excitation energy $E$ is measured relative to Fermi energy $E_{F}$.

Considering an electron for spin $\sigma$ incident on the interface at $x=0$ from left-hand $\mathrm{S}$ at angle $\theta$ to the interface normal, there are four possible trajectories: normal reflection (NR) $\left(b_{\sigma}\right)$, Andreev reflection (AR) $\left(a_{\bar{\sigma}}\right)[32]$, transmission to the right-hand electrode as an electronlike quasiparticle (ELQ) $\left(c_{\sigma}\right)$ and as a holelike quasiparticle 
(HLQ) $\left(d_{\bar{\sigma}}\right)$. For the parallel configuration, from the Eq. (4), the solutions to the $\mathrm{BdG}$ equation in the junction regions are, respectively,

$$
\begin{gathered}
\Psi_{L}(x)=\left(\begin{array}{c}
u_{L+} \mathrm{e}^{i \phi_{L+}} \\
v_{L+}
\end{array}\right) \mathrm{e}^{i k_{+}^{e} x \cos \theta}+a_{\bar{\sigma}}\left(\begin{array}{c}
v_{L+} \mathrm{e}^{i \phi_{L+}} \\
u_{L+}
\end{array}\right) \mathrm{e}^{i \bar{k}_{+}^{h} x \cos \theta}+ \\
+b_{\sigma}\left(\begin{array}{c}
u_{L_{-}} \mathrm{e}^{i \phi_{L-}} \\
v_{L_{-}}
\end{array}\right) \mathrm{e}^{-i \bar{k}_{-}^{e} x \cos \theta}
\end{gathered}
$$

for $x<0$; and

$$
\Psi_{R}(x)=c_{\sigma}\left(\begin{array}{c}
u_{R+} \mathrm{e}^{i \phi_{R+}} \\
v_{R+}
\end{array}\right) \mathrm{e}^{i k_{+}^{e} x \cos \theta}+d_{\bar{\sigma}}\left(\begin{array}{c}
v_{R-} \mathrm{e}^{i \phi_{R-}} \\
u_{R-}
\end{array}\right) \mathrm{e}^{-i k_{-}^{h} x \cos \theta}
$$

for $x>0$, where $a_{\bar{\sigma}}, b_{\sigma}, c_{\sigma}$ and $d_{\bar{\sigma}}$ corresponding, respectively, to coefficients for the Andreev reflection, normal reflection, transmission to the right $d$-wave $\mathrm{S}$ as electrolike quasiparitcles and transmission as holelike quasiparticles. The wave vectors of the electrolike and holelike quasiparticles in the $d$-wave $\mathrm{S}$ are given by

$$
k_{+}^{e}=\sqrt{2 m\left(E_{F}+\sqrt{E^{2}-\left|\Delta_{+}\right|^{2}}\right)} / \hbar
$$

and

$$
\bar{k}_{-}^{e}=\sqrt{2 m\left(E_{F}+\sqrt{E^{2}-\left|\Delta_{-}\right|^{2}}\right)} / \hbar
$$

are the wave vectors for the electronlike quasiparticles corresponding to two different transport directions,

$$
k_{-}^{h}=\sqrt{2 m\left(E_{F}-\sqrt{E^{2}-\left|\Delta_{-}\right|^{2}}\right)} / \hbar
$$

and

$$
\bar{k}_{+}^{h}=\sqrt{2 m\left(E_{F}-\sqrt{E^{2}-\left|\Delta_{+}\right|^{2}}\right)} / \hbar
$$

are the wave vectors for the holelike quasiparticles corresponding to two different transport directions. The Bogoliubov amplitudes read

$$
\begin{aligned}
& \left(u_{L(R) \pm}\right)^{2}=\left\{1+\sqrt{1-\left|\Delta_{ \pm}\right|^{2} / E^{2}}\right\} / 2 \\
& \left(v_{L(R) \pm}\right)^{2}=\left\{1-\sqrt{1-\left|\Delta_{ \pm}\right|^{2} / E^{2}}\right\} / 2 \\
& \exp \left[i \phi_{L(R) \pm}\right]=\frac{\Delta_{L(R) \pm}}{\left|\Delta_{L(R) \pm}\right|} \exp \left[i \phi_{L(R)}\right] .
\end{aligned}
$$

All the coefficients in Eqs. (5) and (6) can be determined by boundary condition at $x=0$. The matching conditions for the wave function are given by $\Psi_{\mathrm{II}}(0)=\Psi_{\mathrm{I}}(0)$, $\left(d \Psi_{\mathrm{II}} / d x\right)_{x=0}-\left(d \Psi_{\mathrm{I}} / d x\right)_{x=0}=2 m U \Psi_{\mathrm{I}}(0) / \hbar^{2}$, we obtain

$$
a_{\bar{\sigma}}=\Theta_{1} / \Theta_{2}
$$

$$
\begin{aligned}
& \Theta_{1}=\left[z_{1}^{2}+\left(z_{2}+i h_{1}\right)^{2}\right] \times \\
& \times\left[u_{R+} u_{R-} u_{L+} v_{L-} \mathrm{e}^{i\left(\phi_{R+}+\phi_{L+}\right)}+v_{R+} v_{R-} v_{L+} u_{L-} \mathrm{e}^{i\left(\phi_{R-}+\phi_{L^{-}}\right)}\right]- \\
& -\left[1+z_{1}^{2}+\left(z_{2}+i h_{1}\right)^{2}\right] \times \\
& \times\left[u_{R+} u_{R-} v_{L+} u_{L-} \mathrm{e}^{i\left(\phi_{R+}+\phi_{L_{-}}\right)}+u_{L+} v_{L_{-}} v_{R+} v_{R-} \mathrm{e}^{i\left(\phi_{R-}+\phi_{L+}\right)}\right]+ \\
& +u_{L+} u_{L-} u_{R-} v_{R+} \mathrm{e}^{i\left(\phi_{L+}+\phi_{L_{-}}\right)}+v_{L+} v_{L_{-}} v_{R-} u_{R+} \mathrm{e}^{i\left(\phi_{R+}+\phi_{R-}\right)}- \\
& -2\left(z_{2}+i h_{1}\right)^{2} \times \\
& \times\left[u_{R+} u_{R-} v_{L+} u_{L_{-}} \mathrm{e}^{i\left(\phi_{R+}+\phi_{L^{-}}\right)}-u_{L_{+}} v_{L_{-}} v_{R+} v_{R-} \mathrm{e}^{i\left(\phi_{R-}+\phi_{L+}\right)}\right] \text {, }
\end{aligned}
$$

$$
\Theta_{2}=\left[1+z_{1}^{2}+\left(z_{2}+i h_{1}\right)^{2}\right] \times
$$$$
\times\left[u_{R+} u_{R-} u_{L+} u_{L-} \mathrm{e}^{i\left(\phi_{R+}+\phi_{L-}\right)}+v_{R+} v_{R-} v_{L+} v_{L-} \mathrm{e}^{i\left(\phi_{R-}+\phi_{L+}\right)}\right]-
$$

$$
-\left[z_{1}^{2}+\left(z_{2}+i h_{1}\right)^{2}\right] \times
$$$$
\times\left[u_{R+} u_{R-} v_{L+} v_{L-} \mathrm{e}^{i\left(\phi_{R+}+\phi_{L+}\right)}+v_{R+} v_{R-} u_{L+} u_{L-} \mathrm{e}^{i\left(\phi_{R-}+\phi_{L-}\right)}\right]-
$$$$
-v_{R+} u_{R-} u_{L-} v_{L+} \mathrm{e}^{i\left(\phi_{L+}+\phi_{L-}\right)}-u_{R+} v_{R-} u_{L+} v_{L-} \mathrm{e}^{i\left(\phi_{R+}+\phi_{R-}\right)}-
$$$$
-2\left(z_{2}+i h_{1}\right)^{2} \times
$$$$
\times\left[v_{R+} v_{R-} v_{L+} v_{L-} \mathrm{e}^{i\left(\phi_{R-}+\phi_{L+}\right)}-u_{R+} u_{R-} u_{L+} u_{L-} \mathrm{e}^{i\left(\phi_{R+}+\phi_{L-}\right)}\right],
$$

where $\phi=\phi_{R}-\phi_{L}, \quad z_{1}=m U_{0} /\left(\hbar^{2} k_{F} \cos \theta\right)=z_{10} / \cos \theta$, $z_{2}=m P /\left(\hbar^{2} k_{F} \cos \theta\right)=z_{20} / \cos \theta, h_{1}=m U_{h} /\left(\hbar^{2} k_{F} \cos \theta\right)=$ $=h_{0} / \cos \theta$. Here, we assume the condition of $E_{F} \gg \Delta(\theta)$. Thus, we have made the approximation of $\bar{k}_{L(R) \pm}^{e(h)}=$ $=k_{L(R) \mp}^{e(h)} \approx k_{F}$. Following obtained $a_{\bar{\sigma}}(E, \phi, \theta)$, the Josephson critical current can be calculated using the generalized coefficient of Andreev reflection $a_{\bar{\sigma}}(E, \phi, \theta)$, which is obtained by analytic continuation of $E$ to $i \omega_{n}, \phi$ to $-\phi$, and $\theta$ to $\pi-\theta$, yielding [33]

$$
\begin{gathered}
I=\frac{e \Delta_{L} k_{B} T}{2 \hbar} \times \\
\times \operatorname{Re} \sum_{\omega_{n}} \int_{-\pi / 2}^{\pi / 2} \frac{a_{\sigma}\left(i \omega_{n}, \phi, \theta\right)-a_{\sigma}\left(i \omega_{n},-\phi, \pi-\theta\right)}{\Omega_{n L}} \cos \theta d \theta,
\end{gathered}
$$

where $\Delta_{L}$ is the order parameter of the left $d$-wave $\mathrm{S}$, $\Omega_{n \sigma}=\left(\omega_{n}^{2}+\Delta_{L}^{2}\right)^{1 / 2}$ and $\omega_{n}=(2 n+1) \pi k_{B} T$ denotes the Matsubara frequency with $n=0, \pm 1, \ldots$ Substituting Eqs. (14)-(16) into Eq. (17), we obtain the Josephson critical current for configuration as for $\alpha=\beta=0$

$$
I=\frac{e \pi k_{B} T \sin \phi}{2 h} \operatorname{Re}\left\{\sum_{\omega_{\mathbf{n}}} \int_{-\pi / 2}^{\pi / 2} g_{1} \cos \theta d \theta\right\},
$$

$$
\begin{gathered}
g_{1}=2 \Delta_{0}^{2} \cos ^{2} 2 \theta\left[\Delta_{d 0}^{2} \cos ^{2} 2 \theta \cos \phi+\left(z_{1}^{2}+\kappa^{2}\right) \Delta_{d 0}^{2} \cos ^{2} 2 \theta+\right. \\
\left.+\left(1+z_{1}^{2}+\kappa^{2}\right)\left(\omega_{n}^{2}+\gamma_{1}^{2}\right)+4 \kappa \omega_{n} \gamma_{1}\right]^{-1 / 2}, \\
\gamma_{1}=\sqrt{\omega_{n}^{2}+\Delta_{d 0}^{2} \cos ^{2} 2 \theta}, \quad \kappa=z_{2}+i h_{1},
\end{gathered}
$$


and for $\alpha=\beta=\pi / 4$

$$
\begin{gathered}
I=\frac{e \pi k_{B} T \sin \phi}{2 h} \operatorname{Re}\left\{\sum_{\omega_{\mathbf{n}}} \int_{-\pi / 2}^{\pi / 2} g_{2} \cos \theta d \theta\right\}, \\
g_{2}=2 \Delta_{d 0}^{2} \sin ^{2} 2 \theta\left[\Delta_{d 0}^{2} \sin ^{2} 2 \theta \cos \phi-\left(z_{1}^{2}+\kappa^{2}\right) \Delta_{d 0}^{2} \sin ^{2} 2 \theta+\right. \\
\left.+\left(1+z_{1}^{2}+\kappa^{2}\right)\left(\omega_{n}^{2}+\gamma_{2}^{2}\right)-4 \kappa \omega_{n} \gamma_{2}\right]^{-1 / 2} \\
\gamma_{2}=\sqrt{\omega_{n}^{2}+\Delta_{d 0}^{2} \sin ^{2} 2 \theta} .
\end{gathered}
$$

Similarly, we obtain the Josephson critical current for the configuration as for $\alpha \neq \beta$, here assume $\alpha=0, \beta=\pi / 4$

$$
\begin{gathered}
I=\frac{e \pi k_{B} T \cos \phi}{2 h} \operatorname{Re}\left\{\sum_{\omega_{\mathbf{n}}-\pi / 2}^{\pi / 2} g_{3} \cos \theta d \theta\right\}, \\
g_{3}=\frac{2 i \gamma_{1} \Delta_{d 0} \sin 2 \theta \cos 2 \theta}{\left(1+z_{1}^{2}+\kappa^{2}\right) \omega_{n}\left(\gamma_{1}+\gamma_{2}\right)-\left(z_{1}^{2}+\kappa^{2}\right) \omega_{n}\left(\gamma_{2}-\gamma_{1}\right)+\lambda} \cdot \frac{1}{\gamma_{1}}, \\
\xi=\sqrt{\omega_{n}^{2}+\Delta_{d 0}^{2} \cos ^{2} 2 \theta} \sqrt{\omega_{n}^{2}+\Delta_{d 0}^{2} \sin ^{2} 2 \theta}, \\
\lambda=i \Delta_{d 0}^{2} \cos 2 \theta \sin 2 \theta \sin \phi+2 \kappa\left(\omega_{n}^{2}+\xi\right) .
\end{gathered}
$$

\section{Results and discussions}

In the following, we will analyze the dc Josephson currents as functions of the temperature and phase. In this paper, we take $\alpha=\beta=0$ or $\pi / 4$ and $\alpha \neq \beta \quad(\alpha=0$, $\beta=\pi / 4)$ in numerical calculations. Let us first discuss the numerical results for the de Josephson currents $I_{c}$ as a function of tempertures for $d_{x^{2}-y^{2}}$ superconductor junction. With the help of Eqs. (14) and (17), the dependence of the critical currents $I_{c}$ on the temperature for the barrier strength $\left(z_{10}\right)$, the roughness strength $\left(z_{20}\right)$ and the exchange field $\left(h_{0}\right)$ at interface are shown in Figs. $2(\alpha=\beta)$ and $3(\alpha \neq \beta)$.

Figure 2 displays the temperature dependence of the critical current in $d / \mathrm{FI} / d$ junctions. They are plotted in Figs. 2,a,b with $\alpha=\beta=0$ and in Figs. 2,c,d with $\alpha=\beta=\pi / 4$. With increasing the barrier strength at interface, Andreev reflection is always suppressed and the dc Josephson critical current in $d_{x^{2}-y^{2}}$-wave $\mathrm{S} / \mathrm{FI} / d_{x^{2}-y^{2}}$ wave $\mathrm{S}$ decreases. It is shown that the junction changes from 0 to $\pi$ states with increasing exchange field at weak barrier $\left(z_{10}=0.5\right)$ by shown in Fig. 2,a, which phenomenon is the same as pointed out by Bergeret et al. in Ref. 18 . The presence of $h_{0}$ usually suppresses the Josephson critical current. However, we notice that the critical current is hanced by the exchange field at strong barrier strength $\left(z_{10}=3.0\right)$ for $0-0$ junction at low temperatures (shown in Fig. 2,b). Meanwhile, behaviors of the critical currents depending on $T$ conform to each other at high temperatures and they have no connection to $h_{0}$. It shows similar

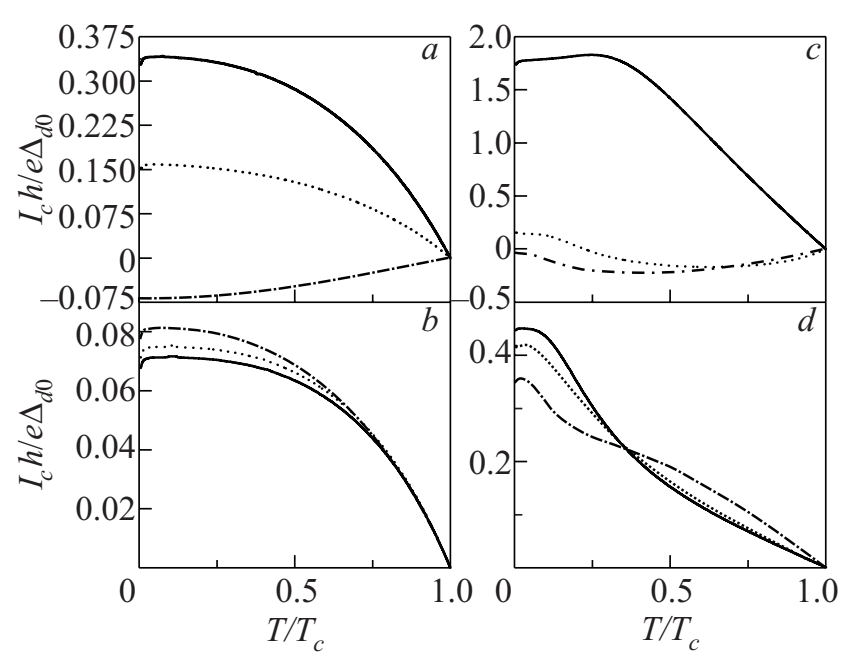

Fig. 2. Dependence of the critical current on $T$ for different the barrier strength and the exchange field at interface: $h_{0}=0$ (solid line), $h_{0}=1.0$ (dot line), $h_{0}=2.0$ (dash-dot line). Here $\phi=\pi / 2$, in which $\alpha=\beta=0$ and $z_{10}=0.5, z_{20}=0.5(a) ; \alpha=\beta=0$ and $z_{10}=3.0, \quad z_{20}=0.5 \quad(b) ; \quad \alpha=\beta=\pi / 4 \quad$ and $\quad z_{10}=0.5$, $z_{20}=0.5(c) ;$ and $\alpha=\beta=\pi / 4$ and $z_{10}=3.0, \quad z_{20}=0.5(d)$.

behavior to that found in the junction of the magnetic barrier by Kashiwaya and Tanaka [24]. It is very interesting that the Josephson current changes its sign with increasing temperature at a certain $h_{0}$, for instance, the junction changes from 0 to $\pi$ states with increasing temperature at $h_{0}=1.0$ (shown in Fig. 2,c). On the other hand, with increasing temperature, $I_{c}$ decreases and drops to zero at $T_{c}$, which expresses the critical temperature of the $d$-wave superconductor. A phenomenon needed to be especially emphasized is that the critical current is also enhanced by exchange field in the case of strong barrier strength $\left(z_{10}=3.0\right)$ and high temperatures for $\pi / 4-\pi / 4$ junction (shown in Fig. 2,d).

In Fig. 3, temperature dependence of the critical current is plotted for junctions with $\alpha \neq \beta \quad(\alpha=0, \beta=\pi / 4)$. It is found that the junction changes from 0 to $\pi$ states with increasing the exchange field whether strong barrier strength or weak one. It is worth pointing out here that $I_{c}$ increases gradually with increasing of the temperature at low temperature and a certain $h_{0}$ (as shown in Fig. 3,b $h_{0}=1.0$ ). When temperature or $h_{0}$ increases, this phenomenon disappears.

Compare the above results, it is found that $I_{c}$ is sensitive to the change of the barrier when $\alpha=\beta=\pi / 4$ or $\alpha \neq \beta$ (shown in Figs. 2,c,d and 3), the amplitude of $I_{c}$ decreases rapidly with increasing of the temperature, that is because of $\Delta_{ \pm} /\left|\Delta_{ \pm}\right|=-1$, ZES are formed at the interface. It is noticeable that the critical current is more sensitive in the case of strong barrier strength than the weak one at low temperature, there is a sudden drop in $I_{c}$ for strong barrier strength at low temperatures (shown in Figs. 2,d and $3, b)$. 


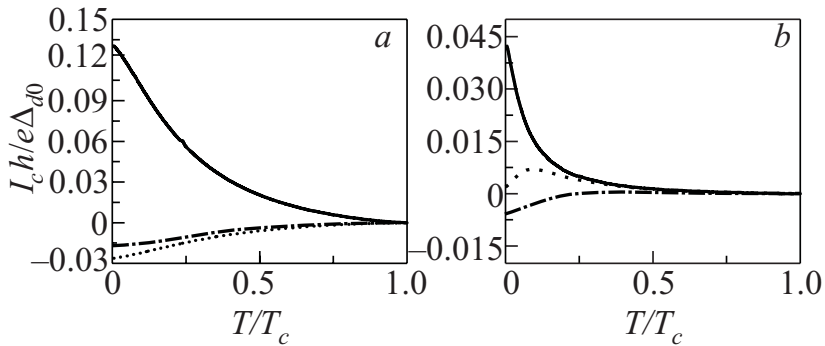

Fig. 3. Dependence of the critical current on $T$ for different the barrier strength and the exchange field at interface: $h_{0}=0$ (solid line), 1.0 (dot line), 2.0 (dash-dot line). Here $\phi=3 \pi / 4$, in which $\alpha=0, \beta=\pi / 4$ and $z_{10}=0.5, z_{20}=0.5(a) ; \alpha=0, \beta=\pi / 4$ and $z_{10}=3.0, z_{20}=0.5(b)$.

Next we study the current-phase relation for $d_{x^{2}-y^{2}}$ superconductor junction. Figures 4 and 5 displays the barrier strength and the roughness strength at interface always suppress the Josephson critical currents. Here, we wish to compare Fig. 4,c with other figures, it is found that, there the exchange field not only could change the junction from 0 to $\pi$ states as shown in Figs. 4, $a$ and $c$ but also could change the period of the oscillation as shown in Fig. 4,c $\left(h_{0}=1.0\right)$. When $\alpha=\beta=0$ or $\pi / 4$, as shown in Fig. 4, the Josephson critical current in a $d / \mathrm{FI} / d$ junction depends on the crystal orientation of the $d$-wave $\mathrm{S}$ and $I(\phi)$ is proportional to $\sin \phi$, the periods of the oscillation curves are $2 \pi$. Particularly, as seen from Fig. 5, when $\alpha \neq \beta$ $(\alpha=0, \beta=\pi / 4)$, the phase dependence of the Josephson critical current $I(\phi)$ between two $d$-wave superconductors is predicted to be $\sin 2 \phi$ and the periods of oscillation curves are $\pi$, Fig. 5 shows almost half periodicity compared to Fig. 4, the period of the oscillation becomes short-
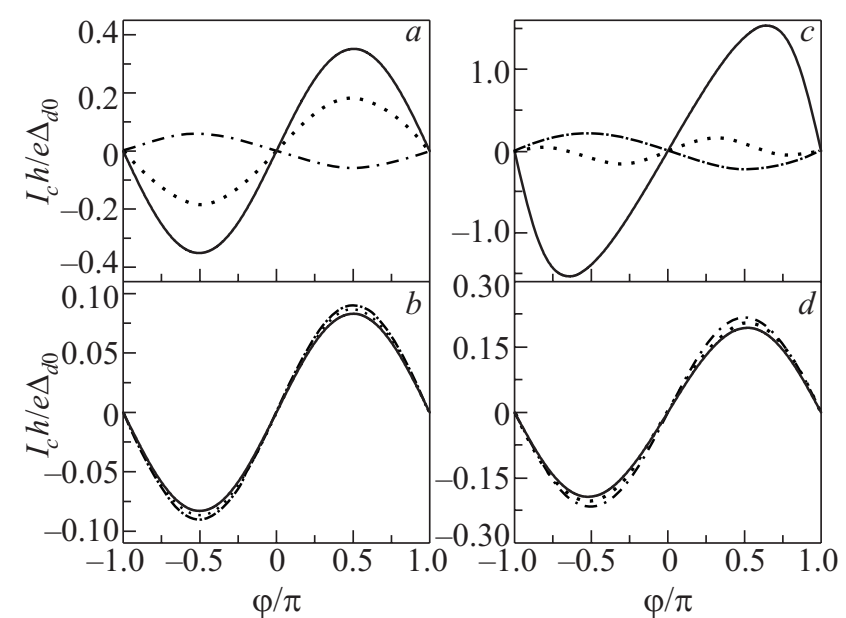

Fig. 4. Dependence of the normalized Josephson current on the phase for different the barrier strength and the exchange field at interface: $h_{0}=0$ (solid line), 1.0 (dot line), 2.0 (dash-dot line). Here $T=0.5 T_{c}$, in which $\alpha=\beta=0$ and $z_{10}=0.5, z_{20}=0.5(a)$; $\alpha=\beta=0$ and $z_{10}=3.0, z_{20}=0.5(b) ; \alpha=\beta=\pi / 4$ and $z_{10}=0.5$, $z_{20}=0.5(c)$; and $\alpha=\beta=\pi / 4$ and $z_{10}=3.0, z_{20}=0.5(d)$.

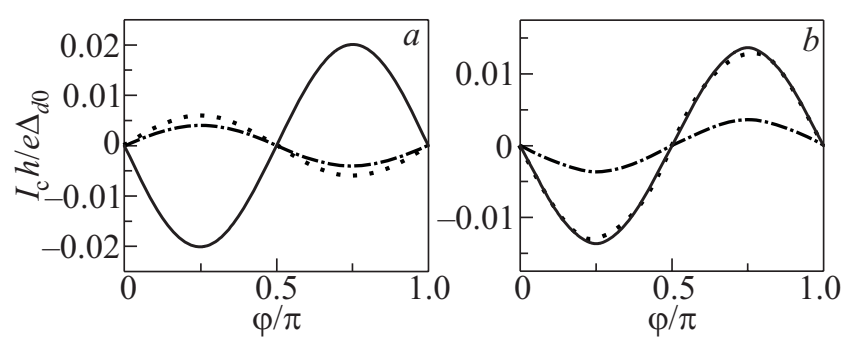

Fig. 5. Dependence of the normalized Josephson current on the phase for different the barrier strength and the exchange field at interface: $h_{0}=0 \quad$ (solid line), 1.0 (dot line), 2.0 (dash-dot line). Here $T=0.5 T_{c}$, in which $\alpha=0, \quad \beta=\pi / 4$ and $z_{10}=0.5$, $z_{20}=0.5(a) ; \alpha=0, \beta=\pi / 4$ and $z_{10}=3.0, z_{20}=0.5(b)$.

er for $\alpha=\beta=0$ or $\pi / 4$. These phenomena are accord with the Ref. 18.

\section{Conclusion}

In this paper, we have studied the temperature dependence of the critical current and the current-phase relation at different the barrier strength and the exchange field at interface. It is demonstrated that the barrier strength and the roughness strength at interface always suppress the critical currents in a $d$-wave $\mathrm{S} / \mathrm{FI} / d$-wave $\mathrm{S}$ junction. Under the condition of strong barrier strength, low temperatures and $\alpha=\beta=0$, the exchange field can contribute to increasing critical current. Meanwhile, the exchange field can change the period of the oscillation, resulting in the current sign and the ground-state transition from 0 to $\pi$. These results are similar to those of Ref. 14, 18, and 21.

However, some different phenomena are found from Ref. 14, mainly located in the condition whether high temperatures or low temperatures which the exchange field can enhance the critical current. Under the condition of strong barrier strength and $\alpha=\beta=0$, the exchange field could enhance the critical currents at low temperatures, moreover, behaviors of the critical currents depending on temperature conform to each other at high temperatures (as shown in Fig. 2,b). Under the condition of strong barrier strength and $\alpha=\beta \neq 0$, the exchange field can contribute to increasing critical current at high temperatures (as shown in Fig. 2,d). An important manifestation of this physical phenomenon is that the critical current increases gradually with increasing the temperature at low temperatures at a certain $h_{0}\left(h_{0}=1.0\right)$. When the temperature increases or $h_{0}$ changes, this phenomenon disappears (as shown in Fig. 3,b). It is expected that the theoretical results obtained here may be further confirmed in the future experiment.

\section{Acknowledgments}

This work is supported by the Program for Excellent Talents in Huangshi Institute of Technology. 
1. B.D. Josephson, Phys. Lett. 1, 251 (1962).

2. A.A. Golubov, M.Y. Kapriyanov, and E. Il'ichev, Rev. Mod. Phys. 76, 411 (2004).

3. B. Kastening, D.K. Morr, D. Manske, and K. Bennemann, Phys. Rev. Lett. 96, 047009 (2006).

4. Y.A. Kolesnichenko, A.N. Omelyanchouk, and S.N. Shevchenko, Fiz. Nizk. Temp. 30, 288 (2004) [Low Temp. Phys. 30, 213 (2004)].

5. Ofer Yuli, Itay Asulin, Gad Koren, and Oded Millo, Phys. Rev. B81, 024516 (2010).

6. A.F. Santander-Syro, M. Ikeda, T. Yoshida, A. Fujimori, K. Ishizaka, M. Okawa, S. Shin, R.L. Greene, and N. Bontemps, Phys. Rev. Lett. 106, 197002 (2011).

7. D.J. Van Harlingen, Rev. Mod. Phys. 67, 515 (1995).

8. C.C. Tsuei and J.R. Kirtley, Rev. Mod. Phys. 72, 969 (2000).

9. Y.A. Kolesnichenko, A.N. Omelyanchouk, and A.M. Zagoskin, Fiz. Nizk. Temp. 30, 714 (2004) [Low Temp. Phys. 30, 535 (2004)].

10. G. Wendin and V.S. Shumeiko, Fiz. Nizk. Temp. 33, 957 (2007) [Low Temp. Phys. 33, 724 (2007)].

11. S. Kawabata, Y. Asano, Y. Tanaka, A.A. Golubov, and S. Kashiwaya, Phys. Rev. Lett. 104, 117002 (2010).

12. F.S. Bergeret, A.F. Volkov, and K.B. Efetov, Rev. Mod. Phys. 77, 1321 (2005).

13. F.S. Bergeret, A.F. Volkov, and K.B. Efetov, Phys. Rev. Lett. 86, 3140 (2001).

14. T. Yokoyama, Y. Tanaka, and A.A. Golubov, Phys. Rev. B75, 094514 (2007).

15. D. Samal and P.S. Anil Kumar, J. Appl. Phys. 109, 07E129 (2011).

16. A.I. Buzdin, Rev. Mod. Phys. 77, 935 (2005).
17. Y. Rahnavard, G. Rashedi, and T. Yokoyama, J. Phys.: Condens. Matter 22, 415701 (2010).

18. Y. Tanaka and S. Kashiwaya, J. Phys. Soc. Jpn. 69, 1152 (2000).

19. R. Mahmoodi, S.N. Shevchenko, and Yu.A. Kolesnichenko, Fiz. Nizk. Temp. 28, 262 (2002) [Low Temp. Phys. 28, 184 (2002)].

20. S. Kawabata, Y. Asano, Y. Tanaka, A.A. Golubov, and S. Kashiwaya, Phys. Rev. Lett. 104, 117002 (2010).

21. F.S. Bergeret, A.F. Volkov, and K.B. Efetov, Phys. Rev. B64, 134506 (2001).

22. Y. Sawa, T. Yokoyama, Y. Tanaka, and A.A. Golubov, Phys. Rev. B75, 134508 (2007).

23. C.R. Hu, Phys. Rev. Lett. 72, 1526 (1994).

24. S. Kashiwaya and Y. Tanaka, Rep. Prog. Phys. 63, 1641 (2000).

25. S. Kashiwaya, Y. Tanaka, M. Koyanagi, H. Takashima, and K. Kajimura, Phys. Rev. B51, 1350 (1995).

26. Z.C. Dong, D.Y. Xing, Z.D. Wang, Ziming Zheng, and Jinming Dong, Phys. Rev. B63, 144520 (2001).

27. A. Furusaki and M. Tsukada, Solid State Commun. 78, 299 (1991).

28. Y.H. Liao, J. Li, and F. Wang, Mod. Phys. Lett. B25, 2 (2011).

29. Z.C. Dong, D.Y. Xing, Z.D. Wang, and L. Sheng, Z. Phys. B: Condens. Matter 100, 329 (1996).

30. Y. Tanaka and S. Kashiwaya, Physica C274, 357 (1997).

31. P.G. de Gennes, Supercouductivity of Metals and Alloys, Benjamin, New York (1966).

32. A.F. Andreev, Zh. Eksp. Teor. Fiz. 46, 1823 (1964) [Sov. Phys. JETP 19, 1228 (1964)].

33. Y. Tanaka, Phys. Rev. Lett. 72, 3871 (1994). 